\title{
Régularisation d'un problème d'obstacle bilatéral
}

\author{
B. Achachab(*) A. Addou(**), J. Zahi(*) \\ (*)Laboratoire de Modélisation et calcul économique, Université Hassan I, Settat, Maroc. \\ (**)Département de Mathématiques et Informatique, Université Mohammed I, Oujda, Maroc. \\ achchab@yahoo.fr,addou@ @cience.univ-oujda.ac.ma, zahi_ja@yahoo.fr
}

RÉSUMÉ. Le but essentiel de ce travail est la résolution d'un problème d'obstacle bilatéral à l'aide d'une méthode de régularisation .

ABSTRACT. The main objective of this work is the resolution of an bilateral obstacle problem by means of the regularization method.

MOTS-CLÉS : régularisation, inéquation variationnelle, obstacle, problème, bilatéral.

KEYWORDS : regularization, variational inequality, obstacle, problem, bilateral. 


\section{Introduction}

Soient $\Omega$ un domaine borné de $\mathbf{R}^{n}$ de frontière $\Gamma=\partial \Omega$ assez régulière, $g \in$ $H^{1 / 2}(\Gamma)$ et $\psi_{1}, \psi_{2} \in H^{1}(\Omega)$, tels que

$$
\operatorname{Tr}\left(\psi_{1}\right) \leq g \leq \operatorname{Tr}\left(\psi_{2}\right) \text { sur } \Gamma .
$$

On considère le problème d'inéquation variationnelle - dit problème d'obstacle bilatéral - :

Trouver

$$
u \in K=\left\{v \in H^{1}(\Omega): v=g \text { sur } \partial \Omega, \psi_{1} \leq v \leq \psi_{2} \text { p.p. dans } \Omega\right\}
$$

telle que

$$
\int_{\Omega} \nabla u \nabla(v-u)+\langle f, v-u\rangle \geq 0 \forall v \in K
$$

où $f \in L^{2}(\Omega)$.

Il est bien connu que ce problème admet une solution unique (voir D. Kinderlehrer et G. Stampacchia).

On suppose que $\Delta \psi_{1}, \Delta \psi_{2} \in L^{2}(\Omega)$, avec ces hypothèses, nous pouvons écrire un autre problème d'inéquation variationnelle sans contraintes, équivalent à (1) - (2).

Ce problème èquivalent fait intervenir un terme non differentiable. Notre idée de régularisation consiste à remplacer ce terme par un autre qui est differentiable, on obtient ainsi une famille de problèmes d'équations aux dérivées partielles dont la suite des solutions converge vers la solution du problème initial.

Par la méthode de dualité par les fonctions conjuguées (voir I. Ekeland, R. Temam) on fournit une estimation a-posteriori de l'erreur qui est desirée pour l'implementation pratique de la méthode de régularisation. 


\section{Reformulation et régularisation}

\subsection{Reformulation de (1)-(2)}

Soit $\Omega$ un domaine borné de $\mathbb{R}^{n}$, de frontière $\Gamma=\partial \Omega$ assez régulière. Soient $f \in L^{2}(\Omega), g \in H^{1 / 2}(\Gamma), \psi_{1}, \psi_{2} \in H^{1}(\Omega)$, qu'on suppose vérifier :

$$
\begin{gathered}
\psi_{1} \leq g \leq \psi_{2} \text { p.p. sur } \Gamma \\
\Delta \psi_{1}, \Delta \psi_{2} \in L^{2}(\Omega)
\end{gathered}
$$

On notera :

$$
F=f-\Delta \psi_{2} \in L^{2}(\Omega)
$$

On définit : $F^{+}=\max \{F, 0\}, F^{-}=\max \{0,-F\}$

et $\psi=\psi_{1}-\psi_{2}$.

Soit le convexe :

$$
K=\left\{v \in H_{g}^{1}(\Omega): \psi_{1} \leq v \leq \psi_{2} \text { p.p. } \operatorname{sur} \Omega\right\}
$$

On considère le problème d'inéquation variationnelle -dit problème d'obstacle bilatéral- :

$$
\left(P_{\psi_{1,2}}\right)\left\{\begin{array}{l}
\text { Trouver } u \in K \\
a(u, v-u)+\langle f, v-u\rangle \geq 0 \quad \text { pour tout } v \in K
\end{array}\right.
$$

où $a(.,$.$) est défini par$

$$
a(u, v)=\int_{\Omega} \nabla u . \nabla v, \quad u, v \in H^{1}(\Omega) .
$$

Il est bien connu que le problème $\left(P_{\psi_{1,2}}\right)$ admet une solution unique (voir D. Kinderlehrer et G. Stampacchia).

Dans toute la suite on utilisera la même notation $g$ pour designer un élément de $H^{1 / 2}(\Gamma)$ et un élément de $H^{1}(\Omega)$ dont la trace sur $\Gamma$ est $g$, de même par $k=g-\psi_{1} / \Gamma$, on désignera aussi un élément $k$ de $H^{1}(\Omega)$ dont la trace sur $\Gamma$ est $k$.

On va écrire le problème d'obstacle $\left(P_{\psi_{1,2}}\right)$ sous une nouvelle forme. 


\section{Théorème 1 :}

$u$ est solution du problème $\left(P_{\psi_{1,2}}\right)$ si et seulement si $w=u-\psi_{1}$ est solution du problème d'inéquation variationnelle suivant :

$(P)\left\{\begin{array}{l}\text { Trouver } w \in H_{k}^{1}(\Omega) \\ a(w, v-w)+\varphi(v)-\varphi(w)+\left(H^{-}, v-w\right) \geq 0 \text { pour tout } v \in H_{k}^{1}(\Omega)\end{array}\right.$

où $\varphi$ est la fonctionnelle définie par

$$
\varphi(v)=\left(F^{-},(v+\psi)^{-}\right)+\left(H^{+}, v^{+}\right) \quad v \in H_{k}^{1}(\Omega),
$$

et $H=F^{+}-\Delta \psi$

Preuve :(voir A. Addou, E.B. Mermri)

\subsection{Régularisation de $(P)$}

L'expression de la fonctionnelle $\varphi$ nous fait penser à prendre $\varphi_{\varepsilon}$ sous la forme suivante :

$$
\varphi_{\varepsilon}(v)=\int_{\Omega} H^{+} \phi_{\varepsilon}(v)+F^{-} \psi_{\varepsilon}(v+\psi),(\varepsilon>0, \text { destiné à tendre vers zero }) .
$$

Or $\phi_{\varepsilon}$ et $\psi_{\varepsilon}$ sont deux fonctions définies de $\mathbf{R}$ vers $\mathbf{R}$, dérivables et convexes vérifiant :

$$
\lim _{\varepsilon \rightarrow 0} \phi_{\varepsilon}(t)=t^{+} \quad \text { et } \quad \lim _{\varepsilon \rightarrow 0} \psi_{\varepsilon}(t)=t^{-}
$$

\section{Definition 1 :}

Le problème régularisé de $(P)$ est :

$$
\left(P_{\varepsilon}\right)\left\{\begin{array}{l}
w_{\varepsilon} \in H_{k}^{1}(\Omega) \\
a\left(w_{\varepsilon}, v-w_{\varepsilon}\right)+\varphi_{\varepsilon}(v)-\varphi_{\varepsilon}\left(w_{\varepsilon}\right)+\left\langle H^{-}, v-w_{\varepsilon}\right\rangle \geq 0 \quad \forall v \in H_{k}^{1}(\Omega)
\end{array}\right.
$$

le problème $\left(P_{\varepsilon}\right)$ admet une solution unique (voir D. Kinderlehrer et G. Stampacchia).

A présent on peut proposer les trois exemples suivants de $\varphi_{\varepsilon}$ et $\psi_{\varepsilon}$ qui vérifient les conditions ci-dessus :

$$
\begin{gathered}
\phi_{\varepsilon}^{1}(t)= \begin{cases}t-\frac{\varepsilon}{2} & \text { si } t \geq \varepsilon \\
\frac{t^{2}}{2 \varepsilon} & \text { si } 0 \leq t \leq \varepsilon \\
0 & \text { si } t \leq 0 .\end{cases} \\
\phi_{\varepsilon}^{2}(t)= \begin{cases}t & \text { si } t \geq \varepsilon \\
\frac{1}{2}\left(\frac{t^{2}}{\varepsilon}+\varepsilon\right) & \text { si } 0 \leq t \leq \varepsilon \\
\frac{\varepsilon}{2} & \text { si } t \leq 0 .\end{cases}
\end{gathered}
$$




$$
\begin{gathered}
\phi_{\varepsilon}^{3}(t)= \begin{cases}\sqrt{t^{2}+\varepsilon^{2}} & \text { si } t \geq 0 \\
\varepsilon & \text { si } t \leq 0 .\end{cases} \\
\psi_{\varepsilon}^{1}(t)= \begin{cases}0 & \text { si } t \geq 0 . \\
\frac{t^{2}}{2 \varepsilon} & \text { si }-\varepsilon \leq t \leq 0 . \\
-t-\frac{\varepsilon}{2} & \text { si } t \leq-\varepsilon .\end{cases} \\
\psi_{\varepsilon}^{2}(t)= \begin{cases}0 & \text { si } t \geq 0 \\
\frac{1}{2}\left(\frac{t^{2}}{\varepsilon}+\varepsilon\right) & \text { si }-\varepsilon \leq t \leq 0 \\
-t & \text { si } t \leq-\varepsilon .\end{cases} \\
\psi_{\varepsilon}^{3}(t)= \begin{cases}\varepsilon & \text { si } t \geq 0 \\
\sqrt{t^{2}+\varepsilon^{2}} & \text { si } t \leq 0 .\end{cases}
\end{gathered}
$$

Pour établir la convergence de la suite $w_{\varepsilon}$ nous avons besoin nous avons besoin des résultats suivants (voir R. Glowinski, J.L. Lions, R. Trémolières ) :

Lemme 1 Soit $V$ un espace de Hilbert, $a: V \times V \rightarrow R$ une forme bilinéaire continue et $V$-elliptique, $j: V \rightarrow R$ une fonction faiblement continue convexe positive et $f$ une forme linéaire, continue dans $V$.

On suppose que $j_{\varepsilon}: V \rightarrow R,(\varepsilon>0)$, une famille de fonctions convexes semicontinues inférieurement (s.c.i.) et positives vérifiant :

$$
j_{\varepsilon}(v) \rightarrow j(v) \quad \forall v \in V
$$

$$
u_{\varepsilon} \rightarrow u \text { faiblement dans } V \text { alors on a } j(u) \leq \lim _{\varepsilon \rightarrow 0} \inf j_{\varepsilon}\left(u_{\varepsilon}\right) .
$$

Soient $u$ et $u_{\varepsilon}$ les solutions respectivement des inéquations variationnelles suivantes :

$$
\begin{gathered}
a(u, v-u)+j(v)-j(u)+\langle f, v-u\rangle \geq 0, \quad \forall v \in V \\
a\left(u_{\varepsilon}, v-u_{\varepsilon}\right)+j_{\varepsilon}(v)-j_{\varepsilon}\left(u_{\varepsilon}\right)+\left\langle f, v-u_{\varepsilon}\right\rangle \geq 0, \quad \forall v \in V
\end{gathered}
$$

alors on a $u_{\varepsilon} \rightarrow u$ dans $V$ quand $\varepsilon \rightarrow 0$.

Lemme 2 Soient

$$
j(v)=\int_{\Omega} \phi(v) d x, j_{\varepsilon}(v)=\int_{\Omega} \phi_{\varepsilon}(v) d x
$$

$\mathrm{Si}$

$$
\phi_{\varepsilon}(t) \rightarrow \phi(t) \text { uniformément en } t, \text { quand } \varepsilon \rightarrow 0,
$$

alors (6) et (7) sont vérifiés. 
Lemme 3 :

On a :

$$
\left|\phi_{\varepsilon}(t)-\phi(t)\right| \leq c_{1} \varepsilon \text { et }\left|\psi_{\varepsilon}(t)-\psi(t)\right| \leq c_{2} \varepsilon \quad \forall t \in R, .
$$

pour $c_{1}, c_{2}$ deux constantes indépendantes de $\varepsilon$.

\section{Proposition 1 :}

Soit $w_{\varepsilon}($ resp $w)$ la solution de $\left(P_{\varepsilon}\right)($ resp $(P))$, alors on $a$ : $\left(w_{\varepsilon}\right)_{\varepsilon}$ converge vers $w$ dans $H^{1}(\Omega)$.

\section{Preuve :}

Puisque les $\phi_{\varepsilon}, \psi_{\varepsilon}$ vérifient l'inégalité (9), alors d'aprés les Lemmes 1 et 2, on a la convergence $w_{\varepsilon} \rightarrow w$ quand $\varepsilon \rightarrow 0$ dans $H^{1}(\Omega)$.

Prenant $v=w_{\varepsilon}(\operatorname{resp} v=w)$ dans l'inéqualité du problème $(P)\left(\operatorname{resp}\left(P_{\varepsilon}\right)\right)$, on obtient :

$$
a\left(w-w_{\varepsilon}, w-w_{\varepsilon}\right) \leq \varphi\left(w_{\varepsilon}\right)-\varphi_{\varepsilon}\left(w_{\varepsilon}\right)+\varphi_{\varepsilon}(w)-\varphi(w) .
$$

alors

$$
\left\|w-w_{\varepsilon}\right\|_{H^{1}(\Omega)}^{2} \leq\left|\varphi\left(w_{\varepsilon}\right)-\varphi_{\varepsilon}\left(w_{\varepsilon}\right)+\varphi_{\varepsilon}(w)-\varphi(w)\right| .
$$

Donc

$$
\left\|w-w_{\varepsilon}\right\|_{H^{1}(\Omega)}^{2} \leq\left|\varphi\left(w_{\varepsilon}\right)-\varphi_{\varepsilon}\left(w_{\varepsilon}\right)\right|+\left|\varphi_{\varepsilon}(w)-\varphi(w)\right| .
$$

D'aprés l'inégalité (6), on a :

$$
\left\|u-u_{\varepsilon}\right\|_{H^{1}(\Omega)}^{2} \leq 2 \int_{\Omega}\left(F^{-} c_{1}+H^{+} c_{2}\right) \varepsilon d x .
$$

Par conséquent, on obtient l'éstimation a priori suivante :

$$
\left\|u-u_{\varepsilon}\right\|_{H^{1}(\Omega)} \leq\left(\int_{\Omega} 2\left(F^{-} c_{1}+H^{+} c_{2}\right)\right)^{\frac{1}{2}} \sqrt{\varepsilon} d x
$$




\section{Estimation a-posteriori de l'erreur}

Dans cette section nous allons utiliser la dualité par les fonctions conjuguées afin de déterminer une estimation a-posteriori de l'erreur de la solution du problème approché $\left(P_{\varepsilon}\right)$. Nous avons besoin des résultats préliminaires suivants (voir I. Ekeland, R. Temam ) :

Soient $V$ et $V^{*}$ (resp $Y$ et $Y^{*}$ ) deux espaces vectoriels topologiques mis en dualité par la forme bilinéaire $\langle., .\rangle_{V}\left(\operatorname{resp}\langle., .\rangle_{Y}\right)$.

Soit $\varphi$ une fonction de $V$ dans $\overline{\mathbf{R}}=\mathbf{R} \cup\{-\infty,+\infty\}$, sa fonction conjuguée est définie par

$$
\varphi\left(v^{*}\right)=\sup _{v \in V}\left\langle v^{*}, v\right\rangle_{V}-\varphi(v), \quad v^{*} \in V^{*} .
$$

On suppose qu'il existe un opérateur linéaire $L$ continu de $V$ dans $Y, L \in \mathcal{L}(V, Y)$, de transposé $L^{*} \in \mathcal{L}\left(Y^{*}, V^{*}\right)$.

Soit $J$ une fonction de $V \times Y$ dans $\overline{\mathbf{R}}$, on considère le problème de minimisation suivant :

$$
u \in V, J(u, L u)=\inf _{v \in V} J(v, L v)
$$

La fonction conjuguée de $J$ est donnée par

$$
J^{*}\left(y^{*}, v^{*}\right)=\sup _{v \in V, y \in Y}\left\{\left\langle v^{*}, v\right\rangle_{V}+\left\langle y^{*}, y\right\rangle_{Y}-J(v, y)\right\}
$$

\section{Théorème 2 :}

Soient $V$ est un espace de Banach réflexif et $Y$ un espace vectoriel normé.

Soit $J: V \times Y \rightarrow \bar{R}$ une fonction strictement convexe s.c.i. propre et vérifiant :

(i) $\exists u_{0} \in V$, tel que $J\left(u_{0}, L u_{0}\right)<\infty$ et $y \rightarrow J\left(u_{0}, y\right)$ est continue en L $u_{0}$.

(ii) $J(v, L v) \rightarrow+\infty$, quand $\|v\|_{V} \rightarrow+\infty, v \in V$.

Alors le problème (10) admet une solution unique $u$, et

$$
J(u, L u)=\inf _{v \in V} J(v, L v)=-\sup _{y^{*} \in Y^{*}} J^{*}\left(-y^{*}, L^{*} y^{*}\right) .
$$

Soit $\Omega$ un sous ensemble ouvert de $\mathbf{R}^{n}, g: \Omega \times \mathbf{R}^{n} \rightarrow \mathbf{R}$ une fonction de Carathéodory c.à.d., $\forall s \in \mathbf{R}^{n}, x \rightarrow g(x, s)$ est une fonction mesurable et pour presque tout $x \in \Omega$, La fonction $s \rightarrow g(x, s)$ est continue.

Alors la fonction conjuguée de la fonction

$$
G(v)=\int_{\Omega} g(x, v(x)) d x
$$

(supposée définie sur un espace fonctionnel $V$ ) est

$$
G^{*}\left(v^{*}\right)=\int_{\Omega} g^{*}\left(x, v^{*}(x)\right) d x, \quad \forall v^{*} \in V^{*}
$$


où

$$
g^{*}(x, y)=\sup _{s \in \mathbf{R}^{N}}\{y s-g(x, s)\} .
$$

Pour le problème $(P)$ on prend :

$$
\begin{aligned}
& V=H^{1}(\Omega), \\
& Y=Y^{*}=\left(L^{2}(\Omega)\right)^{n} \times L^{2}(\Omega) \\
& L v=(\nabla v, v) \\
& J(v, L v)=H(v)+G(L v) \\
& H(v)= \begin{cases}0 & \text { si } v=k \text { sur } \Gamma \\
+\infty & \text { ailleurs. }\end{cases} \\
& G(y)=\int_{\Omega} \frac{1}{2}\left|y_{1}\right|^{2}+H^{+} y_{2}^{+}+F^{-}\left(y_{2}+\psi\right)^{-}+H^{-} y_{2} d x
\end{aligned}
$$

où $y=\left(y_{1}, y_{2}\right)$ avec $y_{1} \in\left(L^{2}(\Omega)\right)^{n}$ et $y_{2} \in L^{2}(\Omega)$, de même on utilise cette notation pour $y^{*} \in Y^{*}$.

D'aprés le Théorème 1, la fonctionnelle conjuguée de $J$ est donnée par :

$$
J^{*}\left(-y^{*}, L^{*} y^{*}\right)=H^{*}\left(L^{*} y^{*}\right)+G^{*}\left(-y^{*}\right)
$$

donc le problème $(P)$ peut s'écrire sous la forme (1.10), c'est à dire :

$$
u \in H^{1}(\Omega), \quad J(u, L u)=\inf _{v \in H^{1}(\Omega)} J(v, L v) .
$$

\section{Lemme 4 :}

Pour $y=\left(y_{1}, y_{2}\right)$ avec $y_{1} \in\left(L^{2}(\Omega)\right)^{n}$ et $y_{2} \in L^{2}(\Omega)$, on a :

$$
J^{*}\left(-y^{*}, L^{*} y^{*}\right)= \begin{cases}\leq \int_{\Omega} \frac{1}{2}\left|y_{1}^{*}\right|^{2}+\nabla k y_{1}^{*}+k y_{2}^{*} d x & \text { si }- \text { div } y_{1}^{*}+y_{2}^{*}=0 \text { dans } \Omega \\
\infty & \begin{array}{l}
\text { et } y_{2}^{*} \geq-F^{-}, y_{2}^{*} \geq-H^{+} \\
\text {ailleurs. }
\end{array}\end{cases}
$$

\section{Preuve :}

On a :

$$
\begin{aligned}
H^{*}\left(L^{*} y^{*}\right) & =\sup _{v \in H^{1}(\Omega)}\{\langle L v, y\rangle-H(v)\} \\
& =\sup _{v \in H_{k}^{1}(\Omega)} \int_{\Omega}\left(\nabla v y_{1}^{*}+v y_{2}^{*}\right) d x \\
& =\int_{\Omega}\left(\nabla k y_{1}^{*}+k y_{2}^{*}\right) d x+\sup _{v \in H_{0}^{1}(\Omega)} \int_{\Omega}\left(\nabla v y_{1}^{*}+v y_{2}^{*}\right) d x \\
& = \begin{cases}\int_{\Omega}\left(\nabla k y_{1}^{*}+k y_{2}^{*}\right) d x & \text { si }-d i v y_{1}^{*}+y_{2}^{*}=0 \text { dans } \Omega \\
\infty & \text { ailleurs }\end{cases}
\end{aligned}
$$


et

$$
\begin{aligned}
G^{*}\left(-y^{*}\right) & =\sup _{y \in Y}\left\{\left\langle-y^{*}, y\right\rangle-G(y)\right\} \\
& =\sup _{y \in Y} \int_{\Omega}\left(-y_{1}^{*} y_{1}-y_{2}^{*} y_{2}-\frac{1}{2}\left|y_{1}\right|^{2}-H^{+} y_{2}^{+}-F^{-}\left(y_{2}+\psi\right)^{-}-H^{-} y_{2}\right) d x \\
& = \begin{cases}\leq \int_{\Omega}\left(\frac{1}{2}\left|y_{1}^{*}\right|^{2}\right) d x & \text { si } y_{2}^{*} \geq-F^{-} \text {et } y_{2}^{*} \geq-H^{+} \\
\infty & \text { ailleurs. }\end{cases}
\end{aligned}
$$

Alors

$$
J^{*}\left(-y^{*}, L^{*} y^{*}\right)= \begin{cases}\leq \int_{\Omega} \frac{1}{2}\left|y_{1}^{*}\right|^{2}+\nabla k y_{1}^{*}+k y_{2}^{*} d x & \text { si }-\operatorname{div} y_{1}^{*}+y_{2}^{*}=0 \text { dans } \Omega \\
\infty & \begin{array}{l}
\text { et } y_{2}^{*} \geq-F^{-}, y_{2}^{*} \geq-H^{+} \\
\text {ailleurs. }
\end{array}\end{cases}
$$

\section{Lemme 5 :}

Pour $w_{\varepsilon}$ et $w$ solution de $\left(P_{\varepsilon}\right)$ et $(P)$ respectivement, on $a$ :

$$
\begin{gathered}
\frac{1}{2}\left\|\nabla\left(w_{\varepsilon}-w\right)\right\|_{L^{2}(\Omega)}^{2} \leq \int_{\Omega} \frac{1}{2}\left|\nabla w_{\varepsilon}\right|^{2}+H^{+} w_{\varepsilon}^{+}+F^{-}\left(w_{\varepsilon}+\psi\right)^{-}+H^{-} w_{\varepsilon}+\frac{1}{2}\left|y_{1}^{*}\right|^{2}+\nabla k y_{1}^{*}+k y_{2}^{*} d x . \\
\forall y^{*}=\left(y_{1}^{*}, y_{2}^{*}\right) \in Y^{*}, \text { avec }- \text { div } y_{1}^{*}+y_{2}^{*}=0 \text { et } y_{2}^{*} \geq-F^{-}, y_{2}^{*} \geq-H^{+} \text {dans } \Omega .
\end{gathered}
$$

\section{Preuve :}

On a :

$$
\begin{aligned}
J\left(w_{\varepsilon}, L w_{\varepsilon}\right)-J(w, L w)= & \int_{\Omega} \frac{1}{2}\left|\nabla w_{\varepsilon}\right|^{2}-\frac{1}{2}|\nabla w|^{2}+H^{+} w_{\varepsilon}^{+}-H^{+} w^{+}+F^{-}\left(w_{\varepsilon}+\psi\right)^{-} \\
& -F^{-}(w+\psi)^{-}+H^{-}\left(w_{\varepsilon}-w\right) d x .
\end{aligned}
$$

Utilisons l'inéquation de (P) avec $v=w_{\varepsilon}$, on obtient :

$$
J\left(w_{\varepsilon}, L w_{\varepsilon}\right)-J(w, L w) \geq \frac{1}{2}\left\|\nabla\left(w_{\varepsilon}-w\right)\right\|_{L^{2}(\Omega)}^{2} .
$$

Appliquons le théorème 1 et utilisons le lemme 1, alors on a :

$$
\begin{aligned}
& J\left(w_{\varepsilon}, L w_{\varepsilon}\right)-J(w, L w) \leq \int_{\Omega} \frac{1}{2}\left|\nabla w_{\varepsilon}\right|^{2}+H^{+} w_{\varepsilon}^{+}+F^{-}\left(w_{\varepsilon}+\psi\right)^{-}+H^{-} w_{\varepsilon}+\frac{1}{2}\left|y_{1}^{*}\right|^{2}+\nabla k y_{1}^{*}+k y_{2}^{*} d x . \\
& \forall y^{*}=\left(y_{1}^{*}, y_{2}^{*}\right) \in Y^{*}, \text { avec }-d i v y_{1}^{*}+y_{2}^{*}=0 \text { et } y_{2}^{*} \geq-F^{-}, y_{2}^{*} \geq-H^{+} \text {dans } \Omega .
\end{aligned}
$$




\section{Proposition 2 :}

Si $w_{\varepsilon}$ et $w$ sont les solutions de $\left(P_{\varepsilon}\right)$ et $(P)$ respectivement alors on a l'estimation a-posteriori suivante :

$\frac{1}{2}\left\|\nabla\left(w_{\varepsilon}-w\right)\right\|_{L^{2}(\Omega)}^{2} \leq \int_{\Omega} H^{+} w_{\varepsilon}^{+}+F^{-}\left(w_{\varepsilon}+\psi\right)^{-}-w_{\varepsilon}\left(H^{+} \phi_{\varepsilon}^{\prime}\left(w_{\varepsilon}\right)+F^{-} \psi_{\varepsilon}^{\prime}\left(w_{\varepsilon}+\psi\right)\right)$.

\section{Preuve :}

Puisque $\phi_{\varepsilon}$ est différentiable l'inéquation de $\left(P_{\varepsilon}\right)$ est équivalente à : $w_{\varepsilon} \in H_{k}^{1}(\Omega)$ :

$$
a\left(w_{\varepsilon}, v\right)+\int_{\Omega}\left(H^{+} \phi_{\varepsilon}^{\prime}\left(w_{\varepsilon}\right)+F^{-} \psi_{\varepsilon}^{\prime}\left(w_{\varepsilon}+\psi\right)+H^{-}\right) v d x=0
$$

d'où $w_{\varepsilon}$ vérifie le problème de Dirichlet suivant :

$$
\left\{\begin{array}{l}
-\Delta w_{\varepsilon}+H^{+} \phi_{\varepsilon}^{\prime}\left(w_{\varepsilon}\right)+F^{-} \psi_{\varepsilon}^{\prime}\left(w_{\varepsilon}+\psi\right)+H^{-}=0 \text { dans } \Omega \\
w_{\varepsilon}=k \operatorname{sur} \Gamma
\end{array}\right.
$$

Si on pose

$$
y_{1}^{*}=\nabla w_{\varepsilon} \text { et } y_{2}^{*}=\left(H^{+} \phi_{\varepsilon}^{\prime}\left(w_{\varepsilon}\right)+F^{-} \psi_{\varepsilon}^{\prime}\left(w_{\varepsilon}+\psi\right)+H^{-}\right) w_{\varepsilon} .
$$

alors on a :

$$
-\operatorname{div}_{1}^{*}+y_{2}^{*}=0 \text { et } y_{2}^{*} \geq-F^{-}, y_{2}^{*} \geq-H^{+}
$$

Donc d'aprés le lemme 5 on a une estimation a-posteriori de l'erreur :

$$
\begin{aligned}
\frac{1}{2}\left\|\nabla\left(w_{\varepsilon}-w\right)\right\|_{L^{2}(\Omega)}^{2} \leq & \int_{\Omega} \nabla w_{\varepsilon} \nabla\left(w_{\varepsilon}-k\right)+H^{+} w_{\varepsilon}^{+}+F^{-}\left(w_{\varepsilon}+\psi\right)^{-}+H^{-} w_{\varepsilon} \\
& +k\left(H^{+} \phi_{\varepsilon}^{\prime}\left(w_{\varepsilon}\right)+F^{-} \psi_{\varepsilon}^{\prime}\left(w_{\varepsilon}+\psi\right)+H^{-}\right) .
\end{aligned}
$$

Si on choisit $v=w_{\varepsilon} \in H^{1}(\Omega)$ dans (13) on aura :

$$
a\left(w_{\varepsilon}, w_{\varepsilon}\right)+\int_{\Omega}\left(H^{+} \phi_{\varepsilon}^{\prime}\left(w_{\varepsilon}\right)+F^{-} \psi_{\varepsilon}^{\prime}\left(w_{\varepsilon}+\psi\right)+H^{-}\right) w_{\varepsilon} d x=0
$$

d'où

$$
\int_{\Omega} \nabla w_{\varepsilon} \nabla\left(w_{\varepsilon}-k\right)+\int_{\Omega}\left(H^{+} \phi_{\varepsilon}^{\prime}\left(w_{\varepsilon}\right)+F^{-} \psi_{\varepsilon}^{\prime}\left(w_{\varepsilon}+\psi\right)+H^{-}\right)\left(w_{\varepsilon}-k\right)=0
$$

L'estimation a-posteriori de l'erreur (14), devient :

$$
\frac{1}{2}\left\|\nabla\left(w_{\varepsilon}-w\right)\right\|_{L^{2}(\Omega)}^{2} \leq \int_{\Omega} H^{+} w_{\varepsilon}^{+}+F^{-}\left(w_{\varepsilon}+\psi\right)^{-}-w_{\varepsilon}\left(H^{+} \phi_{\varepsilon}^{\prime}\left(w_{\varepsilon}\right)+F^{-} \psi_{\varepsilon}^{\prime}\left(w_{\varepsilon}+\psi\right)\right) .
$$


Pour le choix (1) et (2) de $\phi_{\varepsilon}$ et $\psi_{\varepsilon}$, l'estimation a-posteriori (12) de l'erreur est :

$$
\frac{1}{2}\left\|\nabla\left(u_{\varepsilon}-u\right)\right\|_{L^{2}(\Omega)}^{2} \leq \int_{\left[0 \leq w_{\varepsilon \leq \varepsilon}\right]} H^{+} w_{\varepsilon}\left(1-\frac{w_{\varepsilon}}{\varepsilon}\right)+\int_{\left[-\varepsilon \leq w_{\varepsilon}+\psi \leq 0\right]} F^{-}\left(w_{\varepsilon}+\psi\right)\left(1-\frac{w_{\varepsilon}+\psi}{\varepsilon}\right) .
$$

Pour le choix (3) de $\phi_{\varepsilon}$ et $\psi_{\varepsilon}$, l'estimation a-posteriori (12) de l'erreur est :

$$
\frac{1}{2}\left\|\nabla\left(u_{\varepsilon}-u\right)\right\|_{L^{2}(\Omega)}^{2} \leq \int_{\left[w_{\varepsilon} \geq 0\right]} H^{+} w_{\varepsilon}\left(1-\frac{w_{\varepsilon}}{\sqrt{w_{\varepsilon}^{2}+\varepsilon^{2}}}\right)+\int_{\left[w_{\varepsilon}+\psi \leq 0\right]} F^{-}\left(w_{\varepsilon}+\psi\right)\left(1-\frac{w_{\varepsilon}+\psi}{\sqrt{\left(w_{\varepsilon}+\psi\right)^{2}+\varepsilon^{2}}}\right) .
$$

Il est facile de voir que le second membre de cette estimation dans les trois cas tend vers 0 lorsque $\varepsilon \rightarrow 0$.

\section{Acknowledgments}

The authors would like to thank the anonymous referees for interesting remarks. This work was supported in part by the Volkswagen Foundation Grant number I/79315.

\section{Bibliographie}

[1] A. Addou, E.B. Mermri, « Sur une méthode de résolution d'un problème d'obstacle. », Math-Recherche \& Applications, $N^{\circ}$ 2, (2000), pp. 59-69,

[2] I. EkLAND, R. TEMAM, « Analyse convexe et problèmes variationnels. », Gauthier-Villars, (1974),

[3] R. Glowinski, J.L. Lions, R. Trémolières, , « Numerical analysis of variatinal inequalities. », North-Holland, Amsterdam, (1981),

[4] W. HAN, J. ZHOU, « The regularization method for an obstacle problem. », Numer. Math. 69, p. 155-166, (1994).

[5] D. Kinderlehrer et G. Stampacchia, « An introduction to variational inequalities and their applications. », Academic Press, (1980). 